\title{
Número de consultas pré-natais associado a fatores maternos e obstétricos em um Centro de Saúde da Família em Sobral (CE)
}

\author{
Number of Prenatal Consultations Associated With Maternal \\ and Obstetric Factors in a Family Health Center
}

Ananda Milena Martins VASCONCELOS $^{\mathrm{I}}$

Galber Santos Oliveira Filho Danielle da Cunha ARaújo ${ }^{I}$

Gabriela Bezerra Cassol ${ }^{1}$ Georgia Velozo Andrade Costa ${ }^{\mathrm{I}}$

Maiany Alves Cisne ${ }^{1}$

Maria Auxiliadora Silva

Oliveira $^{1}$

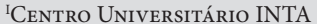

(UNINTA), SOBRAL/CE - BRASIL
ResumoO estudo objetivou analisar o número de consultas pré-natais e idade materna, grau de instrução, estado civil e tipo de parto de gestantes atendidas em um Centro de Saúde da Família em Sobral (CE). Estudo de caráter exploratório, quantitativo, retrospectivo, com análise documental. Foram analisados 64 prontuários de gestantes que realizaram acompanhamento pré-natal no período de 2016 e 2017 em um Centro de Saúde da Família. Foram analisadas as variáveis: número de consultas pré-natais, idade materna, grau de escolaridade, estado civil e tipo de parto. Foi possível observar que 54 gestantes realizaram mais de sete consultas pré-natais. Ademais, nenhuma realizou menos de 4 consultas. 30 gestantes tinham idade entre 21 a 30 anos e realizaram mais de sete consultas, sendo a faixa etária mais predominante e que mais realizou consultas pré-natais. Também pôde-se observar que a maioria das gestantes realizou um número superior de consultas do que é preconizado pelo Ministério da Saúde. Os dados desse estudo indicam uma boa adesão das gestantes ao acompanhamento pré-natal realizado nessa unidade de saúde. Existe uma correlação benéfica entre o número de consultas pré-natais e o tipo de parto realizado. A adesão à realização do pré-natal pode ser influenciada por múltiplos fatores, entre eles: idade materna, escolaridade materna e estado civil. Portanto, as unidades de saúde são responsáveis pela orientação e informação acerca dos benefícios da realização do pré-natal na saúde do binômio mãe-filho.

Palavras-chave: Saúde Materno-infantil; Gravidez; Cuidado Pré-Natal.

ABstraCt The study aimed to analyze the number of prenatal consultations and maternal age, level of education, marital status and type of delivery of pregnant women attended at a Family Health Center Sobral (CE). Exploratory, quantitative, retrospective study with document analysis. It was analyzed 64 medical records of pregnant women who underwent prenatal care in the period 2016 and 2017 in a Family Health Center. The analyzed variables were number of prenatal consultations, maternal age, level of education, marital status and type of delivery. It was possible to observe that 54 pregnant women had more than seven prenatal consultations. Furthermore, none of them had less than 4 con- 
sultations. 30 pregnant women were aged between 21 and 30 years and had more than seven consultations, being the most predominant age group and the one that had more prenatal consultations. It could also be observed that most pregnant women had a higher number of consultations than what is recommended by the Ministry of Health. The data from this study indicate a good adherence of pregnant women to prenatal care carried out in this health unit. There is a beneficial correlation between the number of prenatal visits and the type of delivery performed. Adherence to prenatal care can be influenced by multiple factors, including: maternal age, maternal education and marital status. Therefore, the health units are responsible for providing guidance and information about the benefits of performing prenatal care on the health of the mother-child binomial.

Keywords: Maternal and Child Health; Pregnancy; Prenatal Care.

\section{INTRODUÇÃO}

A realização do acompanhamento pré-natal é uma forma de garantir o bem-estar materno e a saúde do neonato, pois nele é possível prever possíveis complicações físicas e psicológicas além de tratá-las de uma forma segura, por intermédio da realização de procedimentos básicos. Durante a consulta pré-natal são solicitados exames laboratoriais, efetuação de exames clínicos como aferição de pressão arterial, peso, ausculta dos batimentos cardíacos fetais e altura uterina. ${ }^{1}$

No Brasil, o pré-natal tem sido cada vez mais presente na vida das gestantes, mesmo que tardiamente. Dados da pesquisa Nascer revelaram uma cobertura elevada da assistência pré-natal $(98,7 \%)$ tendo $75,8 \%$ das mulheres iniciado o pré-natal antes da $16^{\text {a }}$ semana gestacional e $73,1 \%$ compareceram a seis ou mais consultas. ${ }^{2}$

A adesão de gestantes ao pré-natal se deve ao Programa Nacional de Humanização do Pré-Natal e Nascimento iniciado no ano 2000, o qual apresenta diversos objetivos como estabelecer parâmetros que certifiquem a melhoria do acesso e da qualidade do acompanhamento pré-natal, da assistência ao parto, puerpério e nascimento. ${ }^{3}$

$A$ atenção pré-natal e puerperal possui o objetivo de manutenção e melhoria da saúde materno-infantil por meio de ações de prevenção e promoção da saúde, bem como identificação e tratamento de algum risco gestacional identificado pelo profissional. Portanto, uma assistência pré-natal de qualidade visa proporcionar redução na morbidade e mortalidade materno-infantil. ${ }^{4}$

Quando não há realização do acompanhamento pré-natal a gestante está mais susceptível a eventos adversos durante a gestação e parto, entre eles pré-eclâmpsia, prematuridade e aumento mortalidade neonatal. ${ }^{1}$

Vale ressaltar a importância da realização do pré-natal por todas as gestantes, pois possui abordagem na promoção de saúde, prevenção de agravos, diagnóstico e tratamento de doenças. Ademais, a assistência pré-natal também envolve aspectos psicossociais e educativos. Portanto, tais medidas são eficazes para a redução da morbimortalidade de mães e recém-nascidos. ${ }^{5}$

Diante disso, o estudo objetivou analisar o número de consultas pré-natais e idade 
materna, grau de escolaridade, estado civil e tipo de parto de gestantes atendidas em um Centro de Saúde da Família (CSF) em Sobral, Ceará.

\section{Procedimentos METODOLÓGICOS}

Trata-se de um estudo de caráter exploratório, quantitativo, retrospectivo, com análise documental. Para a realização do estudo utilizou-se como fonte de dados os prontuários impressos de gestantes atendidas em um Centro de Saúde da Família na cidade de Sobral (CE).

A cidade de Sobral fica situada no interior do Ceará, conta com uma população estimada de 205.529 habitantes e possui taxa de mortalidade infantil de 13,25 óbitos por mil nascidos vivos, de acordo com IBGE (IBGE, 2021).

Em 2016, a cidade de Sobral contava com 35 Centros de Saúde da Família, o CSF em que foi realizado o estudo, contava com duas equipes de saúde da família, duas equipes de saúde bucal e um núcleo de apoio à saúde da família (NASF). Os profissionais do CSF realizam atendimentos de pré-natal, puericultura e saúde bucal, bem como ações de promoção da saúde, prevenção, tratamento e reabilitação de doenças.

Foi realizada a análise de 64 prontuários de gestantes que realizaram acompanhamento pré-natal por meio de consultas agendadas com profissionais (médico ou enfermeiro) durante o período de 2016 e 2017 no referido Centro de Saúde da Família. Os prontuários estavam arquivados no Serviço de Arquivo Médico e Estatística (SAME) do CSF. Foram excluídos do estudo aqueles que possuíam ausência de informações acerca das variáveis abordadas.

Aspectos que permitem elaborar a correlação entre fatores maternos e obstétricos foram analisados, tais como: número de consultas pré-natais, idade materna, grau de escolaridade, estado civil e tipo de parto. Para a variante "número de consultas pré-natais", consideraram-se as seguintes eventualidades: de uma a três, de quatro a seis e igual ou mais que sete consultas. Quanto à "idade materna", foram utilizados os seguintes dados: de 15 a 20 , de 21 a 30, de 31 a 40 , 41 e 42 anos. De acordo com "grau de instrução", constataram ensino fundamental, médio e superior. Quanto ao "estado civil", considerou-se com companheiro aquelas com união estável e casadas e as restantes como solteiras. Com relação ao "tipo de parto", verificaram-se números relativos ao parto vaginal e cesáreo.

Para análise dos dados foi utilizado a estatística descritiva com o uso do programa Microsoft Excel, por meio de tabelas compostas pelas variáveis contendo frequências absolutas e relativas.

O presente estudo foi submetido ao comitê de ética da Universidade Estadual Vale do Acaraú (UVA) tendo sido aprovado e obtendo número de parecer $\mathrm{n}^{\circ} 1.878 .614$. Ademais, seguiu os princípios éticos da Resolução $\mathrm{n}^{\circ}$ 466, de 12 de dezembro de 2012, do Conselho Nacional de Saúde que trata sobre pesquisas que envolvem seres humanos.

\section{RESUltados}

Dos 64 prontuários analisados, foi possível observar que 54 gestantes realizaram 
mais de sete consultas pré-natais. Ademais, nenhuma gestante realizou menos de quatro consultas. A tabela 1 apresenta a distribuição do número de consultas pré-natais realizadas por gestantes atendidas no centro de saúde da família.

Tabela 1 - Número de consultas pré-natais realizadas por gestantes atendidas em um Centro de Saúde da Família.

\begin{tabular}{lcc}
\hline \multicolumn{1}{c}{ No. de consultas } & N & \% \\
\hline De $1-3$ & - & - \\
\hline De $4-6$ & 10 & 15,62 \\
\hline$\geq 7$ & 54 & 84,38 \\
\hline
\end{tabular}

Fonte: Produção pelos autores.

Ao verificar o número de consultas pré-natais com a idade da gestante, observou-se que 30 gestantes $(46,88 \%)$ tinham idade entre 21 a 30 anos e realizaram mais de sete consultas sendo a faixa etária mais predominante e que mais realizou consultas pré-natais. Entretanto, apenas uma gestante na faixa etária de 31 a 40 anos de idade realizou de quatro a seis consultas pré-natais, conforme pode ser observado na tabela 2.

Tabela 2 - Número de consultas pré-natais e idade materna de gestantes atendidas em um Centro de Saúde da Família.

\begin{tabular}{ccccccc}
\hline \multirow{2}{*}{$\begin{array}{c}\text { Idade } \\
\text { materna }\end{array}$} & \multicolumn{9}{c}{ De 1-3 } & \multicolumn{7}{c}{ No. de consultas } \\
\cline { 2 - 8 } & $\mathbf{N}$ & $\mathbf{\%}$ & $\mathbf{N}$ & $\mathbf{0}$ & $\mathbf{N}$ & $\underline{\mathbf{0}}$ \\
\cline { 2 - 7 } & - & - & 02 & 3,12 & 11 & 17,19 \\
\hline $15-20$ & - & - & 07 & 10,93 & 30 & 46,88 \\
\hline $21-30$ & - & - & 01 & 1,56 & 11 & 17,19 \\
\hline $31-40$ & - & - & - & - & 02 & 3,13 \\
\hline $41-42$ & & - &
\end{tabular}

Fonte: Produção pelos autores.

Na tabela 3 observa-se o número de consultas pré-natais realizadas pelas gestantes por grau de instrução. Do total de prontuários analisados, 41 gestantes possuíam nível médio completo e realizaram sete consultas ou mais. Ademais, apenas uma mulher possuía nível superior e ela também realizou sete ou mais consultas pré-natais. Vale ressaltar que no prontuário de duas gestantes a informação acerca do grau de instrução estava ausente. 
Tabela 3 - Grau de instrução e número de consultas pré-natais realizadas por gestantes atendidas em um Centro de Saúde da Família.

\begin{tabular}{|c|c|c|c|c|c|c|}
\hline \multirow[t]{3}{*}{ Grau de instrução } & \multicolumn{6}{|c|}{ No. de consultas } \\
\hline & \multicolumn{2}{|c|}{ De 1-3 } & \multicolumn{2}{|c|}{ De 4-6 } & \multicolumn{2}{|c|}{$\geq 7$} \\
\hline & $\mathbf{N}$ & $\%$ & $\mathbf{N}$ & $\%$ & $\mathbf{N}$ & $\%$ \\
\hline Fundamental incompleto & - & & 05 & 7,82 & 11 & 17,18 \\
\hline Médio completo & - & & 04 & 6,26 & 41 & 64,06 \\
\hline Superior completo & - & & - & - & 01 & 1,56 \\
\hline Não responderam & & & 01 & 1,56 & 01 & 1,56 \\
\hline
\end{tabular}

Fonte: Produção pelos autores.

De acordo com os dados da tabela 4, foi possível verificar a relação entre estado civil das gestantes com o número de consultas pré-natais realizadas. Diante disso, 25 gestantes casadas realizaram uma maior quantidade de consultas pré-natais, sendo sete ou mais consultas.

Tabela 4 - Estado civil e número de consultas pré-natais realizadas por gestantes atendidas em um Centro de Saúde da Família.

\begin{tabular}{lcccccc}
\hline \multirow{2}{*}{ Estado civil } & \multicolumn{7}{c}{ No. de consultas } \\
\cline { 2 - 8 } & \multicolumn{2}{c}{ De 1-3 } & \multicolumn{2}{c}{ De 4-6 } & \multicolumn{2}{c}{ 7 } \\
\cline { 2 - 7 } & N & \% & N & \% & N & \% \\
\hline Solteira & - & - & 03 & 4,69 & 11 & 17,18 \\
\hline Casada & - & - & 02 & 3,12 & 25 & 39,06 \\
\hline União estável & - & - & 05 & 7,82 & 18 & 28,13 \\
\hline
\end{tabular}

Fonte: Produção pelos autores.

Ao ser analisado o tipo de parto (tabela 5) observou-se que a maioria dos partos foi cesárea sendo realizados por mulheres que realizaram mais de sete consultas pré-natais.

Tabela 5 - Número de consultas pré-natais associado ao tipo de parto realizadas por gestantes atendidas em um Centro de Saúde da Família

\begin{tabular}{cccccccc}
\hline \multirow{2}{*}{$\begin{array}{c}\text { Tipo de } \\
\text { parto }\end{array}$} & \multicolumn{7}{c}{ No. de consultas } \\
\cline { 2 - 7 } & De 1-3 & N & \% & N & De 4-6 & N & \% \\
\cline { 2 - 7 } & - & - & 04 & 6,25 & 21 & 32,81 \\
\hline Vaginal & - & - & 06 & 9,37 & 33 & 51,57 \\
\hline Cesárea & - & - &
\end{tabular}

Fonte: Produção pelos autores. 


\section{Discussão}

O Ministério da Saúde preconiza a realização de, no mínimo, seis consultas de acompanhamento pré-natal a todas as gestantes, assegurando uma atenção pré-natal e puerperal qualificada e humanizada com ações que integrem todos os níveis de atenção à saúde. ${ }^{7}$

No presente estudo foi observado que a maioria das gestantes $(84,37 \%)$ realizou mais de 7 consultas pré-natais, sendo um número de consultas superior ao preconizado. Desse modo, os dados desse estudo indicam uma boa adesão das gestantes ao acompanhamento pré-natal realizado nessa unidade de saúde.

Os achados evidenciados no presente artigo corroboram com os resultados obtidos em um estudo que apresenta que $89 \%$ das gestantes estudadas realizaram seis consultas ou mais ${ }^{4}$, bem como outra pesquisa com 768 puérperas, demonstrou que $74,7 \%$ realizaram as seis consultas mínimas preconizadas pelo Ministério da Saúde. ${ }^{5}$

Sabe-se que os extremos de idade materna, menor que 17 anos e maior que 35 anos, representam fatores de risco gestacional, pois podem estar associados a problemas frequentes como baixo peso ao nascer, maior risco de internações em Unidades de Terapia Intensiva (UTI) Neonatal e mortalidade infantil e perinatal. ${ }^{8}$

Neste estudo a associação do número de consultas pré-natais realizadas com a idade materna demonstrou que 30 mulheres na faixa etária entre 21 e 30 anos realizaram sete ou mais consultas de pré-natal. Portanto, essa foi a faixa etária mais predominante nos atendimentos.
No que tange ao grau de instrução, a pesquisa aponta que 16 gestantes cursaram o fundamental, sendo cinco $(7,81 \%)$ de quatro a seis consultas e $11(17,18 \%)$ sete ou mais, 45 frequentaram o ensino médio, pertencendo quatro $(6,25 \%)$ de quatro a seis consultas e $41(64,06 \%)$ sete ou mais pré-natais e apenas uma (1,56\%) tem curso superior. Os dados da tabela 5 apontam que o número de gestantes com maior grau de instrução que realizaram sete ou mais consultas é 10 vezes maior do que o número de gestantes que realizaram menos de sete. Já as gestantes com uma menor escolaridade não chegam a realizar mais de três consultas. Isso demonstra que o número de consultas pré-natais está intrinsecamente relacionado ao grau de instrução.

Um estudo realizado pelo Departamento de Medicina da Universidade de Taubaté- Brasil com cerca de 3.843 Declarações de Nascidos Vivos (DNV), documento preenchido logo após todos os nascimentos, apontou que as mães com maior grau de escolaridade tinham uma chance duas vezes maior de realizarem mais de seis consultas, assim prevenindo complicações fetais e maternais. ${ }^{9}$

No tocante ao estado civil das gestantes, o estudo evidencia que a junção casada/união estável formam $67,18 \%$ e as solteiras constituem $17,18 \%$, isso em sete ou mais consultas pré-natais. Mulheres solteiras, geralmente, estão relacionadas a uma maior não realização do pré-natal quando relacionadas às casadas. Isso pode estar relacionado ao apoio psicológico do parceiro em consequência da gravidez, favorecendo a realização do acompanhamento pré-natal. ${ }^{1}$

Em um estudo realizado no Brasil com cerca de 23.940 sujeitos exibe que mulhe- 
res sem companheiro apresentaram menor cobertura pré-natal e início mais tardio da assistência. ${ }^{2}$ Logo, as informações desse estudo indicam uma boa adesão das gestantes com companheiros para a realização do pré-natal nessa unidade de saúde.

Em relação ao tipo de parto realizado, a efetivação de quatro a seis consultas ocorreu em 6,25\% dos partos vaginais e 9,37\% de cesarianas. Já a realização de sete consultas ou mais esteve presente em 32,81\% dos partos vaginais e em $51,56 \%$ dos partos cesáreas. Existe uma correlação benéfica entre o número de consultas pré-natais e o tipo de parto realizado, pois grávidas com propensão à diabetes gestacional, pré-eclâmpsia e hipertensão, se consultam com uma maior regularidade devido a essas ou outras condições de saúde. ${ }^{10}$ Diante disso, são importantes a adesão e o comparecimento em consultas frequentes de forma a prevenir complicações materno-fetais.

Em contrapartida, o Brasil é o segundo país do mundo em número de cesáreas com uma taxa de 55\%. Esse número elevado é contrário em relação à recomendação da Organização Mundial da Saúde (OMS) que é de $10 \%$ a $15 \%$, pois a cesárea só pode ser realizada em situações especificas como as de risco para a saúde da mãe e/ou do bebê. Além disso, as cesáreas estão relacionadas a um maior risco de morbidade e mortalidades maternas e perinatal. ${ }^{11}$

\section{CONSIDERAÇões FINAIS}

A realização do acompanhamento pré-natal é imprescindível para que seja avaliada a saúde da criança e do feto, além de identificar e tratar precocemente agravos na saúde materno-infantil. Para que os riscos na gestação sejam minimizados é necessário que a gestante realize no mínimo seis consultas pré-natais durante a gestação.

Em relação às variáveis observou-se que a maioria das gestantes que faziam acompanhamento pré-natal no CSF em que foi feito o estudo realizou mais de sete consultas, possuíam idade entre 21 e 30 anos, realizaram o ensino médio completo, eram casadas e foram submetidas ao parto cesárea.

A quantidade de consultas realizadas, bem como o início precoce do pré-natal, os acompanhamentos e procedimentos feitos durantes as consultas pré-natais, indicam a qualidade da assistência prestada à gestante em cada unidade de saúde.

Portanto, o número de consultas pré-natais pode ser influenciado por múltiplos fatores, entre eles: idade materna, escolaridade materna e estado civil. Portanto, as unidades de saúde são responsáveis pela orientação e informação acerca dos benefícios da realização do pré-natal na saúde do binômio mãe-filho. 


\section{REFERÊNCIAS}

1. Rosa CQ, Silveira DS, Costa JSD. Fatores associados à não realização de pré-natal em município de grande porte. Rev. Saúde Pública. 2014; 48(6): 977-84

2. Viellas EF, Domingues RMS, Dias MAB, Gama SGN, Theme MM FIlha, Costa JV, et al. Assistência pré-natal no Brasil. Cad. Saúde Pública. 2014; 30(Suppl 1): S85-S100

3. Secretaria de Políticas de Saúde Ministério da Saúde (BR). Programa de Humanização no Pré-Natal e Nascimento. Humanização do parto: humanização no pré-natal e nascimento. Brasília, DF: Ministério da Saúde, 2002.

4. Tomasi E, Fernandes PAA, Fischer T, Siqueira FCV, Silveira DS, Thumé E, et al. Qualidade da atenção pré-natal na rede básica de saúde do Brasil: indicadores e desigualdades sociais. Cad. Saúde Pública. 2017; 33(3): e00195815

5. Mendes RB, Santos JMJ, Prado DS, Gurgel RQ, Bezerra FD, Gurgel RQ. Avaliação da qualidade do pré-natal a partir das recomendações do Programa de Humanização no Pré-natal e Nascimento. Ciênc. saúde coletiva. 2020; 25(3):793-804.

6. IBGE, INSTITUTO BRASILEIRO DE GEOGRAFIA E ESTATÍSTICA. Principais Resultados: Sobral. Disponível em: $<$ https://www.ibge.gov.br/geociencias-novoportal/organi zacao-do-territorio/estrutura-territorial/15761-areas-dos-municipios.html?t=destaques\& $\quad \mathrm{c}=23 \quad 12908>$. Acesso em 28 jul 2021.

7. BRASIL. Pré-natal e puerpério: atenção qualificada e humanizada: manual técnico. Brasília, DF: Ministério da Saúde. Disponível em: https://bvsms.saude.gov.br/bvs/publicacoes/manual_pre_natal_puerperio_3ed.pdf. Acesso em: 29 jul 2021.

8. Chermont AG, Silva EFA, Vieira CC, Souza LEC Filho, Matsumura ESS, Cunha KC. Fatores de risco associados à prematuridade e baixo peso ao nascer nos extremos da vida reprodutiva em uma maternidade privada. REAS. 2020; 39(e-2110), 1-8

9. Haidar FH, Oliveira UF, Nacimento LFC. Escolaridade materna: correlação com os indicadores obstétrico. Cad. Saúde Pública. 2001; 17(4): 1025-9

10. Silva EV, Costa MAA, Almeida KC, Araujo LMB, Amância NFG. Relação do tipo de parto com o perfil epidemiológico da assistência pré-natal e perinatal em um município de Minas Gerais. Rev. Bras. Saude Mater. Infant. 2020; 20(1): 241-7

11. Mandarino NR, Chein MBC, Monteiro FC Júnior, Brito LMO, Lamy ZC, Nina VJS, et al. Aspectos relacionados à escolha do tipo de parto: um estudo comparativo entre uma maternidade pública e outra privada, em São Luís, Maranhão, Brasil. Cad. Saúde Pública. 2009; 25(7): 1587-96

\section{DADOS DOS AUTORES}

\section{Ananda Milena Martins Vasconcelos}

Discente do curso de medicina do Centro Universitário INTA (UNINTA), Sobral/CE - Brasil. anandamilena@hotmail.com

\section{Galber Santos Oliveira Filho}

Discente do curso de medicina do Centro Universitário INTA (UNINTA), Sobral/CE - Brasil. galberfilho@hotmail.com 


\section{Danielle da Cunha Araújo}

Discente do curso de medicina do Centro Universitário INTA (UNINTA), Sobral/CE - Brasil. danicunhaaraujo@hotmail.com

\section{Gabriela Bezerra Cassol}

Discente do curso de medicina do Centro Universitário INTA (UNINTA), Sobral/CE - Brasil. gabrielabezerracassol@gmail.com

\section{Georgia Velozo Andrade Costa}

Discente do curso de medicina do Centro Universitário INTA (UNINTA), Sobral/CE - Brasil. gvacosta@hotmail.com

\section{Maiany Alves Cisne}

Discente do curso de medicina do Centro Universitário INTA (UNINTA), Sobral/CE - Brasil. maianyac@hotmail.com

\section{Maria Auxiliadora Silva Oliveira}

Docente do curso de medicina do Centro Universitário INTA (UNINTA), Sobral/CE - Brasil. myresearchbio@hotmail.com

Submetido em: 5-4-2021

Aceito em: 12-8-2021 